Relations industrielles

Industrial Relations

\title{
Procedures and Policies of the New York State Labor Relations Board, Kurt L. Hanslowe, Cornell University, Ithaca, New York, 1964, 216 pages.
}

\section{Ronald Pleau}

Volume 20, numéro 4, 1965

URI : https://id.erudit.org/iderudit/027636ar

DOI : https://doi.org/10.7202/027636ar

Aller au sommaire du numéro

Éditeur(s)

Département des relations industrielles de l'Université Laval

ISSN

0034-379X (imprimé)

1703-8138 (numérique)

Découvrir la revue

Citer ce compte rendu

Pleau, R. (1965). Compte rendu de [Procedures and Policies of the New York State Labor Relations Board, Kurt L. Hanslowe, Cornell University, Ithaca, New York, 1964, 216 pages.] Relations industrielles / Industrial Relations, 20(4), 722-723. https://doi.org/10.7202/027636ar

Tous droits réservés @ Département des relations industrielles de l'Université Laval, 1965
Ce document est protégé par la loi sur le droit d'auteur. L’utilisation des services d'Érudit (y compris la reproduction) est assujettie à sa politique d'utilisation que vous pouvez consulter en ligne.

https://apropos.erudit.org/fr/usagers/politique-dutilisation/ 
services d'hôtellerie qui peut contribuer au processus de transformation de la théorie de l'organisation selon l'opinion des auteurs cux-mêmes.

Le projet prit naissonce lorsque le viceprésident d'un hôtel américain demanda ò l'un des auteurs de bâtir un plan de recherche concernant les relations humaines dans son entreprise. II était fasciné par l'idée que les relations entre les gens pouvaient être sujettes à une étude. Au début, donc, les auteurs, qui incidemment ont trovaillé ensemble dans cet hôtel pour y étudier les relations inter-personnelles, ne savaient pas quel point ils allaient développer de façon particulière. Aussi le premier chapitre de ce volume est-il consacré à la narration des événements qui ont conduit à l'élaborotion de leur plan de recherche sur le "management » et de la façon dont o débuté leurs trovaux.

Comme dans toute analyse d'un problème, on présente ensuite les caractéristiques de l'entreprise en question et les acteurs y évoluant, en essayant de découvrir où se trouvaient alors les difficultés qui pouvaient être solutionnées.

La première étape à entreprendre pour ces chercheurs consistait donc à clarifier leur rôle, à réorganiser les activités du personnel, à établir leurs relations avec la haute direction et à s'occuper des griefs afin de mettre sur pied leur projet.

Le second développement de cet ouvrage est consacré à la description du travail et à la présentation des résultats de la recherche à mesure qu'elle évoluait dans les différents départements de l'entreprise en question à savoir: le restaurant, la cuisine, le bureau de réception et le bar. De plus, dans I'un des chapitres constituant cette partie, les tâches, les activités, les attitudes, les relations de travail des caissiers et des vérificateurs sont examinées avec soin.

Dans une dernière section on retrouve enfin une évaluation complète de tout ce qui s'est fait, de la stratégie employée, des résultats obtenus et des difficultés rencontrées, pour couronner le tout par un essai d'intégration de cette recherche dans les processus de leodership et de groupe, d'application de la théorie à l'action et de généralisation de cette approche particulière.

Bref, on peut dire que cette étude peut être de lecture intéressante mais ne constitue qu'une approche unique et très personnelle au problème des relations humaines dont on ne pourrait tirer aucun modele d'opplicotion générale.

\section{Ronald Pleau}

\section{Procedures and Policies of the New York State Labor Relations Board, Kurt L. Hanslowe, Cornell University, Ithaca, New York, 1964, 216 pages.}

Ce volume dont l'un des buts est de fournir un guide concernant les procédures des commissions de relations de travail, fut préparé à cause de l'importance toujours grandissante que prend la commission des relations de travail de l'étct de New-York vis-ò-vis les employeurs, les employés et les syndicats et à cause de la rareté des écrits existants en motière de tribunaux administratifs.

Globalement le contenu de cet ouvrage est constitué d'une étude des problèmes d'administration publique et de la loi administrative. On se limite cependant ici au cas de l'étot de New-York dans le champ spécifique des relations patronales-ouvrières.

Comme il se devait, l'auteur nous fait d'abord connaître tous les détails de la loi des relations ouvrières de cet état en attachant une importance toute particulière ò la section 704 de cette dernière qui concerne les protiques de travail interdites. Pour ajouter à la valeur de ce chapitre, il procède ensuite à quelques comparaisons entre cette loi et celle existant au niveau national en s'arrêtant plus longuement sur l'analyse du problème posé par les syndicats de boutique.

C'est à la description de l'organisation et du personnel de la commission des relations de travail qu'est consacré le deuxièrne chapitre dans lequel sont traités des sujets aussi variés mais aussi logiquement reliés que les suivants: la place de la commission dans le département (ou ministère) du travail, la commission des relations de travail et la commission de conciliation, les membres de la commission, et le personnel subordonné.

Ainsi oprès nous avoir présenté les acteurs et leurs instruments de travail, nous passons ò l'examen de leur rôle lorsque l'auteur s'attarde à nous expliquer la procédure antérieur à l'audition formelle pour le cas des pratiques interdites de travail. II soulève ici les problèmes relatifs ou chef d'accusation et à l'enquête informelle menée en ropport avec ce dernier.

Toujours en rapport ovec le même genre de cas, ce sont ensuite les informations concernant l'audition comme telle, la procédure 
subséquente à cette dernière, la décision et l'cuis de la commission, les témoins et les ossignations, bref le stage formel, qui constituent l'objet d'un quatrième chapitre.

D'autre part lorsque l'on procède d̀ l'étude d'un cos de représentation, les procédures varient quelque peu telles les pétitions (chap. 6) les élections et la durée de l'accréditation (chap. 7).

Enfin dans ce qu'on pourrait appeler une troisième section, il est question de la commission dans ses rapports avec les cours de justice (chap. 8), de la juridiction propre de cette commission d'état et de sa relation ovec la commission nationale connue sous le sigle N.L.R.B. (chap. 9). A tout cela s'ajoute toutes les observations aussi pertinentes que possible qu'effectue Hanslowe concernant la réorganisation administrative de la commission des relations de travail de l'étot de New York et les amendements substantiels qui pourraient être adoptés comme règles du système.

Somme toute le présent exposé semble être des plus utiles comme ouvrage de référence pour qui veut trouver tous les détails concernant la législation ouvrière de cet état américain et la façon de procéder des parties constituantes de son système de relations industrielles en ropport avec l'administration de ses règles.

\section{Ronald Pleau}

\section{Agriculture and the Public Interest Toward} a New Form Program, Leon $H$. Keyserling, Conference on Economic Progress, 1001 Connecticut Avenue, N.W., Washington 6, D.C., 1965, 123 pages.

De foçon générale, l'auteur discute du problème américain de la ferme sous ses aspects domestiques et internationaux, surtout dans sa relation avec l'emploi et les autres problèmes de l'économie en général. II fournit nombre de données statistiques et d'analyses nécessaires à de bonnes recom. mandations politiques sur la question.

Por son étude, il entend saisir les éléments qui, selon lui, offrent la plus grande perspective pour la restauration de l'agriculture américaine. II montre comment les programmes proposés sont adéquats d̀ l'intérieur des ressources nationales disponibles et sensibles aux besoins nationaux et mondioux de ce secteur.

L'étude peut servir à démontrer entre outres choses que les fermiers et leurs organisotions devront faire front commun pour résoudre leur problème de chômage, de sousemploi, etc., en mettant au-dessus de celles qui les divisent les choses qui leur permettent de travailler ensemble. Et pour atteindre ce consensus de travail ils devront recourir à l'aide du monde des affaires, du monde du travail, des consommateurs et du gouvernement.

C'est donc la lutte pour aider les fermiers sous-marginaux à s'élever au-dessus de la pouvreté et devenir des gens qui peuvent se suffire à eux-mêmes, pour rendre les fermes de type familial capables de résister aux accrocs de la grande coopérative agricole, et pour faire en sorte que le maximum de ce que peut produire le fermier américain serve à la rescousse de ceux qui sont présentement affligés par un manque de nutrition, qui constitue l'un des principoux sujets traités dans cet ouvrage.

Pour ce faire, Keyserling a donc choisi de développer les thèmes suivants: le déclin du revenu de la ferme, les causes de ce déclin et les mythes qui sont reliés à ces causes de bas revenus, les raisons véritables de ce bas niveau, l'utilisation plus abondante des produits de la ferme à la maison, le problème mondial du marché de l'alimentation.

Puis passant du domaine plus théorique ou domaine pratique, c'est de la rationalisation de la production agricole et de la restauration du revenu de la ferme dont traite l'étude, pour nous fournir finalement un programme assez élaboré de politiques agricoles ovec ses implications et ses coûts.

Tout ou long de son texte, l'auteur vient appuyer ses affirmations par une série de tableaux statistiques sur la situation agricole aux Etats-Unis, lesquels rehaussent d'autant la valeur du contenu de cet ouvrage.

\section{Ronald Pleau}

\section{An International Comparison of Factor Costs and Factor Use, Bagicho Singh Minhas, North-Holland Publishing Com- pany, Amsterdam, 1963, 124 pages.}

Cette étude fait partie d'une série d'ouvroges en rapport avec l'analyse économique, mais le but spécifique de celle que nous vous présentons ici est de considérer les différences entre les coûts relatifs du travail et du capital pour un groupe de pays, et la facilité ou difficulté relative de substituer l'un pour l'autre dons différentes industries. 